\title{
Predicting the UV-vis spectra of oxazine dyes
}

\author{
Scott Fleming, Andrew Mills and Tell Tuttle*
}

\author{
Full Research Paper \\ Address: \\ WestCHEM, Department of Pure and Applied Chemistry, University of \\ Strathclyde, 295 Cathedral Street, Glasgow G1 1XL, UK

\section{Email:} \\ Andrew Mills - a.mills@strath.ac.uk; Tell Tuttle* - \\ tell.tuttle@strath.ac.uk \\ * Corresponding author \\ Keywords: \\ DFT; dyes; oxazine; TD-DFT; UV-vis
}

Open Access

\author{
Beilstein J. Org. Chem. 2011, 7, 432-441. \\ doi:10.3762/bjoc.7.56 \\ Received: 08 November 2010 \\ Accepted: 23 March 2011 \\ Published: 15 April 2011 \\ This article is part of the Thematic Series "Physical organic chemistry". \\ Guest Editor: J. Murphy \\ (C) 2011 Fleming et al; licensee Beilstein-Institut. \\ License and terms: see end of document.
}

\begin{abstract}
In the current work we have investigated the ability of time-dependent density functional theory (TD-DFT) to predict the absorption spectra of a series of oxazine dyes and the effect of solvent on the accuracy of these predictions. Based on the results of this study, it is clear that for the series of oxazine dyes an accurate prediction of the excitation energy requires the inclusion of solvent. Implicit solvent included via a polarizable continuum approach was found to be sufficient in reproducing the excitation energies accurately in the majority of cases. Moreover, we found that the SMD solvent model, which is dependent on the full electron density of the solute without partitioning into partial charges, gave more reliable results for our systems relative to the conductorlike polarizable continuum model (CPCM), as implemented in Gaussian 09. In all cases the inclusion of solvent reduces the error in the predicted excitation energy to $<0.3 \mathrm{eV}$ and in the majority of cases to $<0.1 \mathrm{eV}$.
\end{abstract}

\section{Introduction}

Oxazine dyes are a subclass of quinone imines, which are all based upon the $p$-benzoquinone imine or -diimine scaffold. Other important subclasses within the quinone imines include, the azine dyes and thiazine dyes. The structural relationships described are illustrated in Figure 1 for clarity.

All the dyes are based on an anthracene skeleton in which one carbon is replaced by a nitrogen atom and another by an additional heteroatom such as $\mathrm{N}, \mathrm{O}$, or $\mathrm{S}$ in the central ring.
Although azine dyes have been found to demonstrate solvatochromism [1-3], and many thiazine dyes are metachromatic $[4,5]$, this investigation is limited to the study of oxazine dyes.

Oxazine dyes are useful partly because they exhibit solvatochromism, this makes them sensitive to their surrounding environment, and hence they have been utilized in various applications as molecular probes [6-8]. In the current investigation we focus on the ten oxazine dyes shown in Figure 2, 
<smiles>N=C1C=CC(=N)C=C1</smiles>

$p$-quinonediimine<smiles>N=c1ccc2nc3ccccc3[nH]c-2c1</smiles>

azine dyes<smiles>N=c1ccc2nc3ccccc3oc-2c1</smiles>

oxazine dyes<smiles>N=c1ccc2nc3ccccc3sc-2c1</smiles>

thiazine dyes

Figure 1: Quinone imine structural relationships.

which are readily soluble in aqueous solution, in order to determine the ability of computational methodology to describe the solvent dependence on the absorption maxima.

Solvatochromism in oxazine dyes has been observed and the mechanism(s) explored in previous studies by various authors [9-11]. Most of these investigations have involved measuring experimentally the spectroscopic features of oxazine dyes, upon varying the solvent polarity. However, some attempt has been made to rationalize these observations by a computational study<smiles>CC(=O)Nc1ccc2nc3c4ccccc4c(=N)cc-3oc2c1</smiles><smiles>CCNc1cc2oc3c/c(=[NH+]\CC)c4ccccc4c-3nc2cc1C</smiles>

Resorufin (2)<smiles>COc1ccc2nc3ccc(=O)cc-3oc2c1</smiles><smiles>CC[N+](CC)=c1ccc2nc3c(N)cc(N)c(C)c3oc-2c1</smiles><smiles>N=c1cc2oc3cc(N)ccc3nc-2c2ccccc12</smiles>

Nile blue (8)<smiles>[3H]CN(CC)c1ccc2nc3c4ccccc4c(=[NH2+])cc-3oc2c1</smiles>

Resazurin (4)<smiles>O=c1ccc2[n+]([O-])c3ccc(O)cc3oc-2c1</smiles><smiles>CC[N+](CC)=c1ccc2nc3c(C(N)=O)cc(O)c(O)c3oc-2c1</smiles>

Oxazine 4 (5)<smiles>CCNc1cc2oc3cc(=[NH+]CC)c(C)cc-3nc2cc1C</smiles>

Oxazine 1 (10)

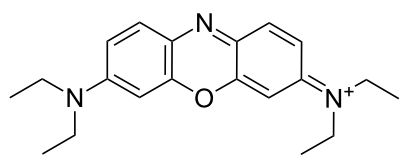

Figure 2: Numbering and structure of oxazine dyes studied in this work (counterions not shown). of the solvatochromism of the oxazine dye Nile red [12]. In the investigation, TD-DFT was applied in order to try and explore the contributing factors in the solvatochromism observed with Nile red, upon gradually increasing the solvent polarity from benzene to acetonitrile. A computational approach such as this is advantageous, because it allows consideration of the individual molecular orbital transitions involved - a feat difficult to achieve via experiment alone.

TD-DFT has become the modern day workhorse for the determination of electronic excited states in medium sized systems (up to 100 atoms) [13-17]. This method performs particularly well for predicting the excitation energies of local excitations and Rydberg states (although in the case of Rydberg states the choice of functional is particularly important with accurate excitation energies for these states requiring large amounts of exact exchange), while its performance in predicting charge-transfer (CT) states is more questionable [18-23]. A number of studies have demonstrated the failures of TD-DFT in predicting CT excitation energies accurately [24-29], while one can also find within the literature examples where TD-DFT performs well for such states [30]. As such in the current work we explore the ability of various density functionals to predict the excitation energies of the intramolecular CT states in a series of oxazine dyes and the effect of solvent models on the accuracy of these predictions. The assessment of these functionals was carried out against the experimental $\lambda_{\max }$ for the absorption of each dye, which was determined experimentally.

\section{Results and Discussion Role of the solvent}

The gas-phase optimized geometries of the dyes were used as the basis for the single point excited state calculations. The six lowest singlet vertical excitation energies and oscillator strengths from the TD-DFT calculations were used to predict the UV-vis spectrum for each dye through the fitting of a Gaussian (with the GaussView default parameters for halfwidth) centered at the computed excitation energies. The predicted UV-vis spectra in the gas-phase, gas//CPCM, and gas//SMD were plotted for each dye, and the $\lambda_{\max }$ values in each case are summarized in Table 1. 
Table 1: Calculated $\lambda_{\max }$ values and the shift resulting from the different solvent models. ${ }^{a}$

\begin{tabular}{crrrrr} 
Dye & \multicolumn{3}{c}{$\lambda_{\text {max }}$} & \multicolumn{3}{c}{ Shift } \\
& Gas $^{\mathrm{b}}$ & CPCM $^{\mathrm{C}}$ & SMD $^{\mathrm{d}}$ & CPCM $^{\mathrm{C}}$ & SMD $^{\mathrm{d}}$ \\
\hline $\mathbf{1}$ & 485 & 548 & 566 & 62 & 81 \\
$\mathbf{2}$ & 458 & 546 & 571 & 88 & 113 \\
$\mathbf{3}$ & 481 & 563 & 587 & 82 & 106 \\
$\mathbf{4}$ & 486 & 570 & 596 & 84 & 110 \\
$\mathbf{5}$ & 468 & 558 & 588 & 90 & 120 \\
$\mathbf{6}$ & 505 & 597 & 626 & 92 & 121 \\
$\mathbf{7}$ & 470 & 596 & 616 & 129 & 146 \\
$\mathbf{8}$ & 512 & 597 & 625 & 85 & 113 \\
$\mathbf{9}$ & 476 & 560 & 584 & 86 & 108 \\
$\mathbf{1 0}$ & 492 & 584 & 617 & 92 & 125 \\
\hline
\end{tabular}

${ }^{\mathrm{a}} \lambda_{\max }$ and the shift are reported in $\mathrm{nm}$. The shifts are reported relative to the gas-phase $\lambda_{\text {max }} \cdot{ }^{b}$ Gas refers to the gas-phase. ${ }^{C} \mathrm{CPCM}$ refers to the TD-DFT single point calculations using the CPCM solvent model on the gas-phase optimized structures. ${ }^{d}$ SMD refers to the TD-DFT single point calculations using the SMD solvent model on the gasphase optimized structures.

Table 2: Magnitudes of dipole and transition moments in Debye. ${ }^{a}$

\begin{tabular}{crrrr} 
& \multicolumn{3}{c}{ Dipole } & \multicolumn{2}{c}{ Transition } \\
Dye & Gas & CPCM & Gas & CPCM \\
& & & & \\
\hline $\mathbf{1}$ & 8.35 & 12.44 & 3.41 & 4.75 \\
$\mathbf{2}$ & 3.72 & 5.21 & 3.28 & 4.66 \\
$\mathbf{3}$ & 3.99 & 5.81 & 3.17 & 4.65 \\
$\mathbf{4}$ & 3.16 & 4.40 & 3.07 & 4.34 \\
$\mathbf{5}$ & 2.27 & 3.10 & 3.69 & 4.91 \\
$\mathbf{6}$ & 3.13 & 4.65 & 3.74 & 5.06 \\
$\mathbf{7}$ & 2.74 & 3.79 & 3.11 & 3.55 \\
$\mathbf{8}$ & 3.24 & 4.94 & 3.59 & 4.98 \\
$\mathbf{9}$ & 11.82 & 16.61 & 3.21 & 4.51 \\
$\mathbf{1 0}$ & 2.16 & 2.99 & 4.00 & 5.19 \\
\hline
\end{tabular}

${ }^{\mathrm{a}} \mathrm{G}$ as refers to the gas-phase. B3LYP TD-DFT calculations and CPCM refers to the solvent phase CPCM/B3LYP TD-DFT calculations. All TD-DFT calculations were carried out on the gas-phase B3LYP/6$311++G(d, p)$ optimized geometries.

The results in Table 1 indicate a red shift of about $60-130 \mathrm{~nm}$ upon moving from the gas-phase to the CPCM solvent description. Upon moving from the CPCM to the SMD description of the solvent we observe a further red shift in the $\lambda_{\max }$ value relative to the gas-phase calculated spectra. This result is consistent with a narrowing of the energy gap between ground and excited states, due to an increased stabilization of the more polarizable excited state by polar solvents. Clearly, the SMD description of the solvent provides greater stabilization of the excited state with the red shift increased on average by $20-30 \mathrm{~nm}$ relative to the spectra obtained within the CPCM solvent model.
The absorbance spectra for oxazine dyes are often described as occurring through CT excitations. Therefore, it is also helpful to examine the dipole moments, and corresponding transition dipole moments in the gas-phase and in the solvent phase. In each case, the transition moments chosen relates to the most significant excited state (vida infra). In the first instance an examination of the dipole and transition moment magnitudes, shows a increase upon moving to the solvated species (Table 2).

The observed increases in magnitude are indicative of polarization by the solvent field. It is also possible to examine the $\mathrm{x}, \mathrm{y}$, and $\mathrm{z}$ components associated with the corresponding dipole and transition moments (Figure 3a and Figure 3b). It should be noted, that the direction associated with each molecule does not change significantly between gaseous and solvated phases, and hence plots are only shown for the latter.

a

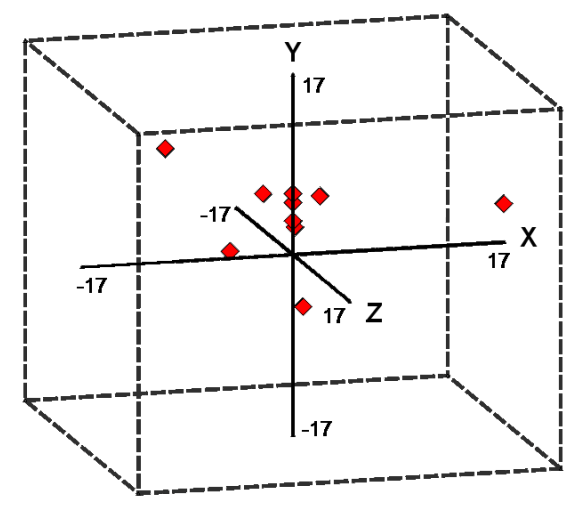

b

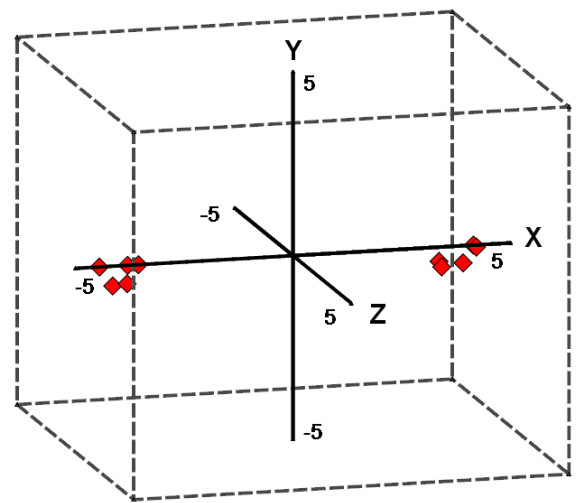

Figure 3: Directions of solvated (a) dipole moments and (b) transition moments from origin $(0,0,0)$

In the above representation the molecule lies in the xy plane, and is elongated along the $\mathrm{x}$-axis, hence the $\mathrm{z}$ component only makes a very slight contribution towards the overall direction. In the case of the ground state dipole moments (Figure 3a), the observed vector differs depending upon the substituents present. For instance, Oxazine 1 (10), Oxazine 4 (5), Resazurin (4), and 
Resorufin (2) have symmetry in the yz plane, and thus have vectors based almost exclusively on the y-axis. In more complex examples, the direction of dipole moment vector is predominately dictated by the positions of the amines/imines, which possess a partial positive charge due to electron donation to the aromatic system. In contrast the transition dipole moments (Figure 3b) show very little variation in the magnitude and direction associated with the transition moment. The only significant contribution lies along the x-axis, and in each case the magnitude is consistently 3-4 Debye. This is indicative of CT along the extended aromatic system and consistent with the classical description of these excitations.

\section{Performance of functionals and solvent models}

The TD-DFT calculations were carried out using the selection of functionals and solvation methods described in the computational methods. The accuracy of the calculated $\lambda_{\max }$ values was assessed against the values obtained experimentally (Table 3).

The data presented in Table 3 clearly shows the important role of the solvent in attaining a realistic description of the excited state. The gas-phase calculated $\lambda_{\max }$ values are all strongly blue shifted relative to the experimental data with an average error of $123 \mathrm{~nm}(0.51 \mathrm{eV})$. The inclusion of the solvent using either of the continuum models (i.e., either the CPCM or SMD model) corrects this error to a large degree with the mean unsigned error (MUE) decreased to $43 \mathrm{~nm}(0.15 \mathrm{eV})$ with the CPCM solvent model and $22 \mathrm{~nm}(0.08 \mathrm{eV})$ within the SMD solvent model at the B3LYP level of theory. Given the large transition dipole moments for the transitions corresponding to the $\lambda_{\max }$ excitations (Table 2), we examined whether a number of functionals that have been shown to perform well for CT states could improve upon the TD-B3LYP calculated excitation energies.

Within the SMD solvent model, TD-B3LYP performs well across all of the dyes. However, the largest errors in the calculated $\lambda_{\max }$ values are found for dyes $\mathbf{1}$ (Darrow red), which is overestimated by $64 \mathrm{~nm}(-0.28 \mathrm{eV})$; and 9 (Celestine blue), which is underestimated by $62 \mathrm{~nm}(0.20 \mathrm{eV})$. In the case of 1 the best performing functional is M06-2X, which still overestimates the value of $\lambda_{\max }(30 \mathrm{~nm} ;-0.14 \mathrm{eV})$ but to a lesser extent relative to B3LYP. However, across the series of dyes, M06-2X is the worst performing functional with an MUE of $33 \mathrm{~nm}$ $(0.11 \mathrm{eV})$ and has the largest error for $9(89 \mathrm{~nm} ; 0.31 \mathrm{eV})$. In contrast, the M06-L functional provides the most accurate representation of 9 , underestimating the $\lambda_{\max }$ excitation by $40 \mathrm{~nm}(0.13 \mathrm{eV})$, however, offers no improvement in the prediction of the $\lambda_{\max }$ value of 1 (M06-L error: $69 \mathrm{~nm}$; $-0.30 \mathrm{eV}$ ). Surprisingly, the gas-phase calculated value of $\lambda_{\max }$ for $\mathbf{1}$ is relatively accurate. The gas-phase B3LYP calculation of 1 underestimates the value of $\lambda_{\max }$ by only $16 \mathrm{~nm}(0.08 \mathrm{eV})$, although this appears to be a fortuitous error cancellation as the solvent calculations systematically result in a strong red shift, which leads to the overestimation mentioned above.

Despite the difficulties associated with predicting the excitation energies of $\mathbf{1}$ and $\mathbf{9}$, the range of different functionals that were tested perform remarkably well once the effect of solvent is taken into account. This is clearly seen in the plot of the errors for the various methodological combinations (Figure 4). Given the non-linear relationship between the observed wavelength

\begin{tabular}{|c|c|c|c|c|c|c|c|c|}
\hline Dye & Exp. & B3LYPb & B3LYPC & B3LYPd & CAM-B3LYPd & $M 06^{d}$ & M06- $L^{d}$ & $M 06-2 X^{d}$ \\
\hline 1 & 502 & 486 & 548 & 566 & 535 & 565 & 571 & 532 \\
\hline 2 & 572 & 458 & 546 & 571 & 581 & 578 & 554 & 578 \\
\hline 3 & 588 & 481 & 563 & 587 & 573 & 591 & 582 & 570 \\
\hline 4 & 602 & 486 & 570 & 596 & 591 & 601 & 578 & 583 \\
\hline 5 & 616 & 468 & 558 & 588 & 580 & 592 & 579 & 576 \\
\hline 6 & 620 & 505 & 597 & 626 & 610 & 629 & 622 & 607 \\
\hline 7 & 624 & 467 & 596 & 616 & 592 & 613 & 625 & 590 \\
\hline 8 & 636 & 512 & 597 & 625 & 608 & 625 & 621 & 607 \\
\hline 9 & 646 & 474 & 560 & 584 & 560 & 586 & 606 & 557 \\
\hline 10 & 654 & 492 & 584 & 617 & 606 & 616 & 606 & 606 \\
\hline MSE & & -123 & -34 & -8 & -22 & -6 & -12 & -25 \\
\hline MUE & & 123 & 43 & 22 & 31 & 23 & 26 & 33 \\
\hline
\end{tabular}

${ }^{a} A l l$ wavelengths are given in nm. MSE is the mean signed error and MUE is the mean unsigned error relative to the experimental $\lambda_{\text {max }}{ }^{b} \mathrm{Gas}-$ phase TD-B3LYP/6-311++G(d,p). ${ }^{c}$ CPCM/TD-B3LYP/6-311++G(d,p). ${ }^{d}$ SMD/TD-DFT/6-311++G(d,p). All single point TD-DFT calculations employed the gasphase optimized structures. 


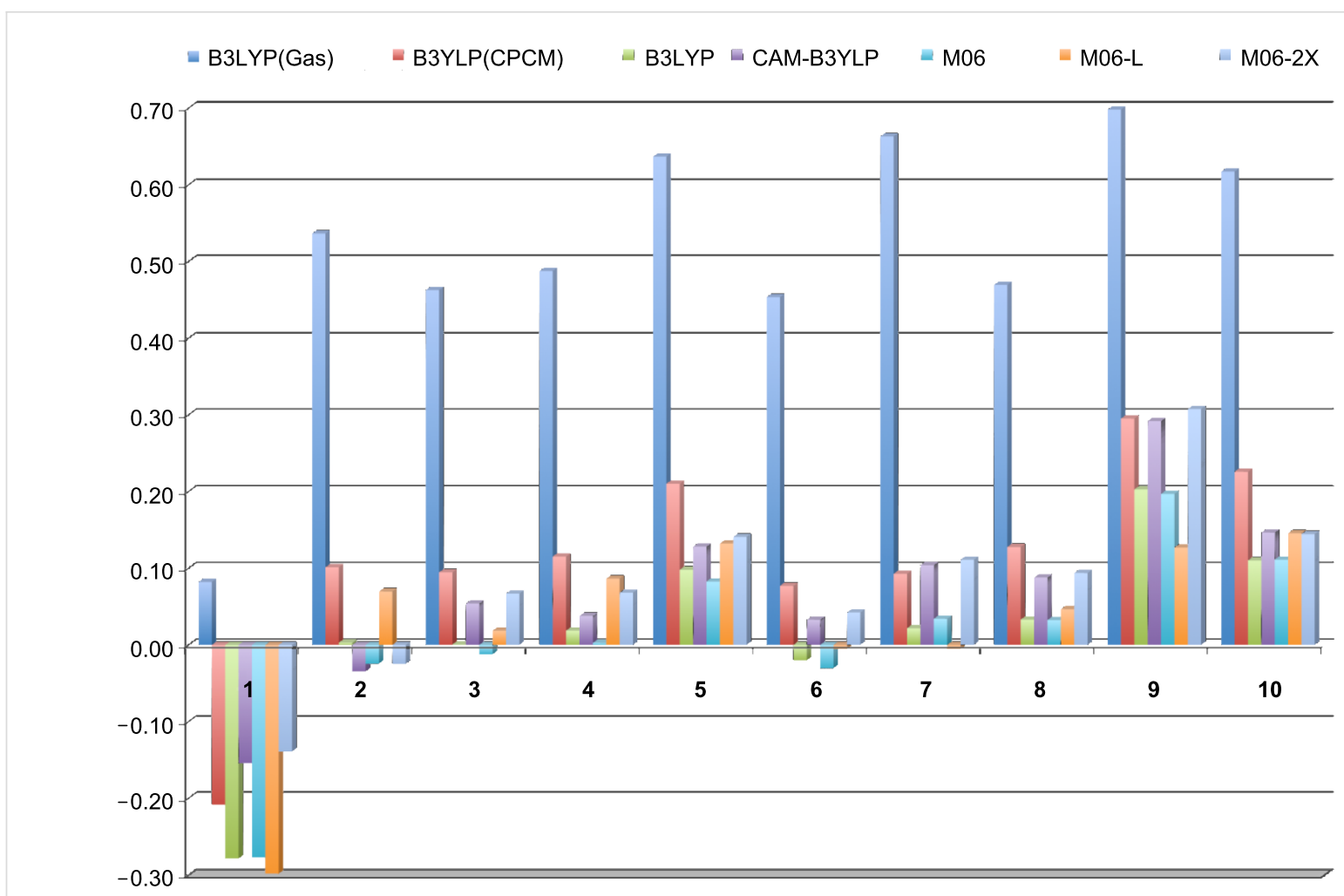

Figure 4: Error between experimental and calculated $\lambda_{\max }$ values for each dye at the different levels of theory investigated. All errors are reported in eV. All structures were optimized in the gas-phase at the B3LYP/6-311++G(d,p) level of theory. B3LYP(Gas) refers to the TD-B3LYP calculation in the gas-phase. B3LYP(CPCM) refers to the TD-B3LYP calculation within the CPCM solvent model. All other TD-DFT calculations were carried out using the SMD solvent model as described in the computational methods.

and the excitation energy (i.e., an error at a high excitation energy will have a lesser impact on the calculated $\lambda_{\max }$ than an equally sized error at a lower excitation energy), the errors associated with the computed excitation energies at the various levels of theory, shown in Figure 4, are in $\mathrm{eV}$. Increasing the percentage of $\mathrm{HF}$ exchange is considered beneficial for lowlying excited states that have an ionic character and as such suffer, to a greater extent, from a self-interaction error. This error can be corrected to some extent by increasing the percentage of HF exchange [19]. However, in our dyes, this was not observed, as both the CAM-B3LYP (greater HF exchange at long range) and M06-2X functionals produce larger errors (Figure 4). Clearly in the series of dyes examined, the increased HF exchange leads to a slight over-correction, which has also been observed by others [22].

The calculated transition dipole moments for the various dyes are consistent with a CT nature of the excitation. Moreover, the largest errors are obtained for $\mathbf{1}$ and $\mathbf{9}$, which also have significantly larger ground state dipole moments, relative to the other dyes. TD-DFT is well-known to fail in a variety of CT excitations, which is in contrast to the results obtained for the other eight dyes. Therefore, we employed the Tozer diagnostic to examine whether the calculated excitation energies indeed correspond to CT excitations from an orbital overlap perspective.

\section{Orbital overlap}

Within the literature there are conflicting cases as to the accuracy of TD-DFT in predicting CT states [24-30]. In some cases, TD-DFT methods appear to perform reasonably well, whereas in other cases - generally long-range CT - TD-DFT significantly underestimates the excitation energy. In an effort to help identify those excitations where TD-DFT is likely to fail, Tozer and co-workers have recently introduced the use of an orbital overlap diagnostic which utilizes the spatial overlap of the unperturbed ground state orbitals in order to assess the likelihood of an accurate TD-DFT excitation energy, for local, Rydberg and intramolecular CT excitations between those orbitals.

The diagnostic, $\Lambda$, considers the spatial overlap between the orbitals involved in the excitation. Where more than one set of orbitals contribute to the excitation (as commonly occurs in TD-DFT calculations) the orbital overlaps are scaled by the contribution $(\kappa)$ from each pair. In the following, we 


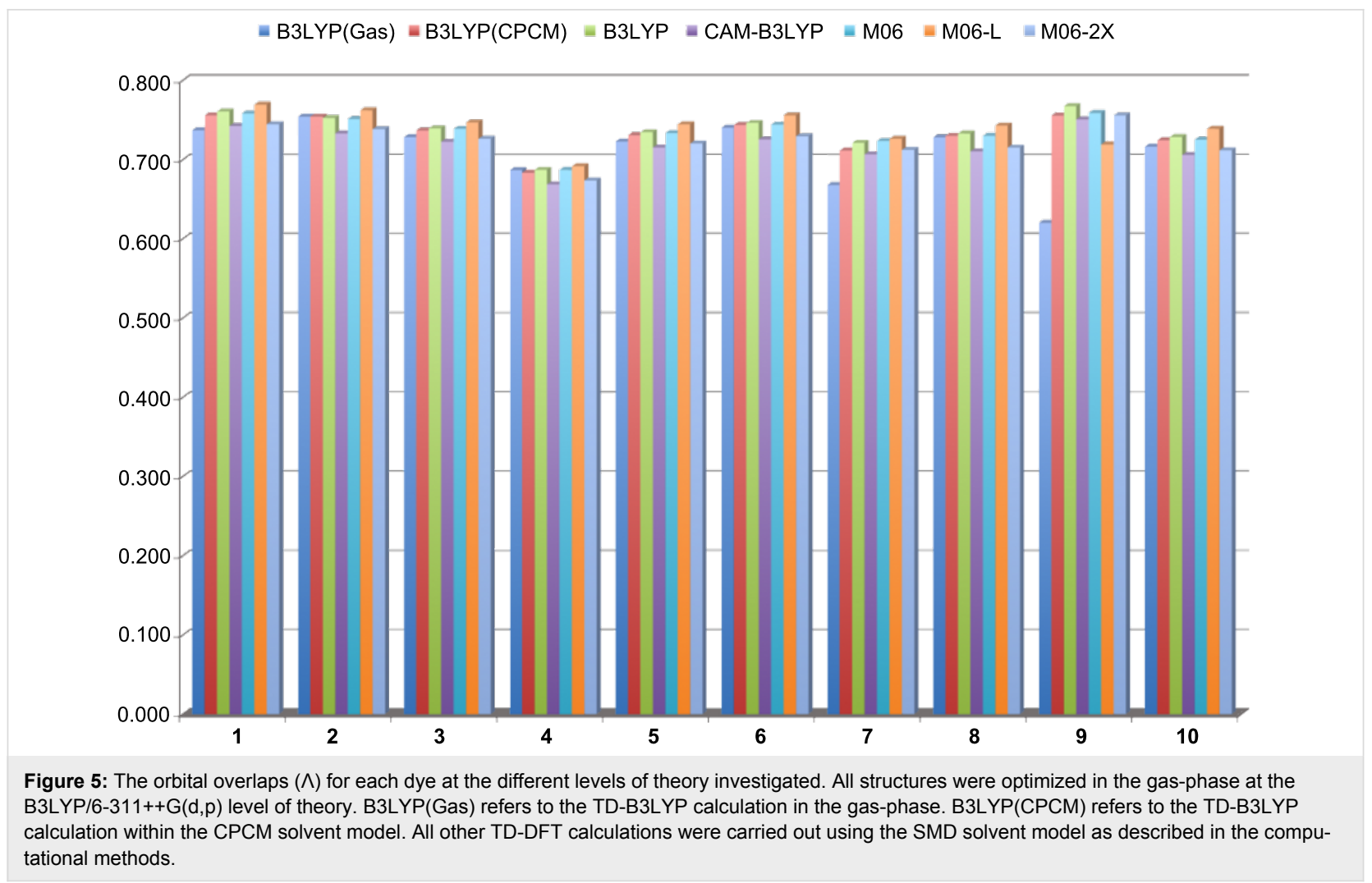

have employed the same form of $\Lambda$ as that introduced by Tozer to investigate the spatial overlap between our orbital pair, namely:

$$
\Lambda=\frac{\sum_{\mathrm{i}, \mathrm{a}} \kappa_{\mathrm{ia}}^{2} \mathrm{O}_{\mathrm{ia}}}{\sum_{\mathrm{i}, \mathrm{a}} \kappa_{\mathrm{ia}}^{2}}
$$

where the spatial overlap $\left(\mathrm{O}_{\mathrm{ia}}\right)$ between the occupied orbital $\left(\varphi_{\mathrm{i}}\right)$ and the virtual orbital $\left(\varphi_{\mathrm{a}}\right)$ is given by the inner product of the moduli of the two orbitals:

$$
\mathrm{O}_{\mathrm{ia}}=\int\left|\varphi_{\mathrm{i}}(r)\right|\left|\varphi_{a}(r)\right| \mathrm{d} r
$$

and $\kappa_{\mathrm{ia}}$ is the largest coefficient in the CI expansion for each orbital pair.

The resulting overlaps calculated at the various level of theory for each dye are plotted in Figure 5. The value of $\Lambda$ is largely conserved across the different methods for each dye. In the case of 9 , where there is some variation between the values of $\Lambda$ calculated in either the gas or solvent phase, this is due primarily to the difference in the two orbital pairs that contribute to the $\lambda_{\max }$ excitation. For the primary excitation in 9, the $\kappa_{\mathrm{ia}}$ value for the minor contributing orbital pair (HOMO-
3-LUMO in the gas-phase and HOMO-1-LUMO in the solvent phase) varies between $0.4-0.6$, while the major contribution (HOMO-LUMO) remains constant across the series, resulting in the slight variation in the $\Lambda$ values observed for this dye. Figure 5 illustrates that the orbital overlap for the solvent phase calculations is largely unaffected by the choice of functional. This is due primarily to the fact that the predominant contribution to the excitation energy and the nature and overlap of the orbital pair (HOMO-LUMO) is conserved across the different methods. In the gas-phase TD-DFT calculations the number of orbital pairs contributing towards the $\lambda_{\max }$ excitation varies with respect to the solvent calculations - two orbital pairs contribute in the gas-phase calculations for $\mathbf{2}, \mathbf{3}, \mathbf{5}, 7, \mathbf{9}$, and $\mathbf{1 0}$ - however, a comparable $\Lambda$ value is obtained in most cases due to the dominance of the HOMO-LUMO contribution in these excitations as well.

The strong overlap between the occupied and virtual orbital can intuitively be seen by visualizing the orbitals. Given the consistency of the calculated overlaps the orbital contributions to the $\lambda_{\max }$ for each dye across the series of functionals, only the orbitals calculated at the CPCM/B3LYP level of theory are displayed in Table 4.

Tozer and co-workers suggest that an overlap of $\Lambda \leq 0.3$, indicates that TD-DFT will struggle to predict correctly the excita- 
Table 4: Orbital pairs involved in the $\lambda_{\max }$ excitation for each dye. ${ }^{a}$

Dye

2

3

4

5

6
Occupied $\mathrm{MO}(\mathrm{s})$
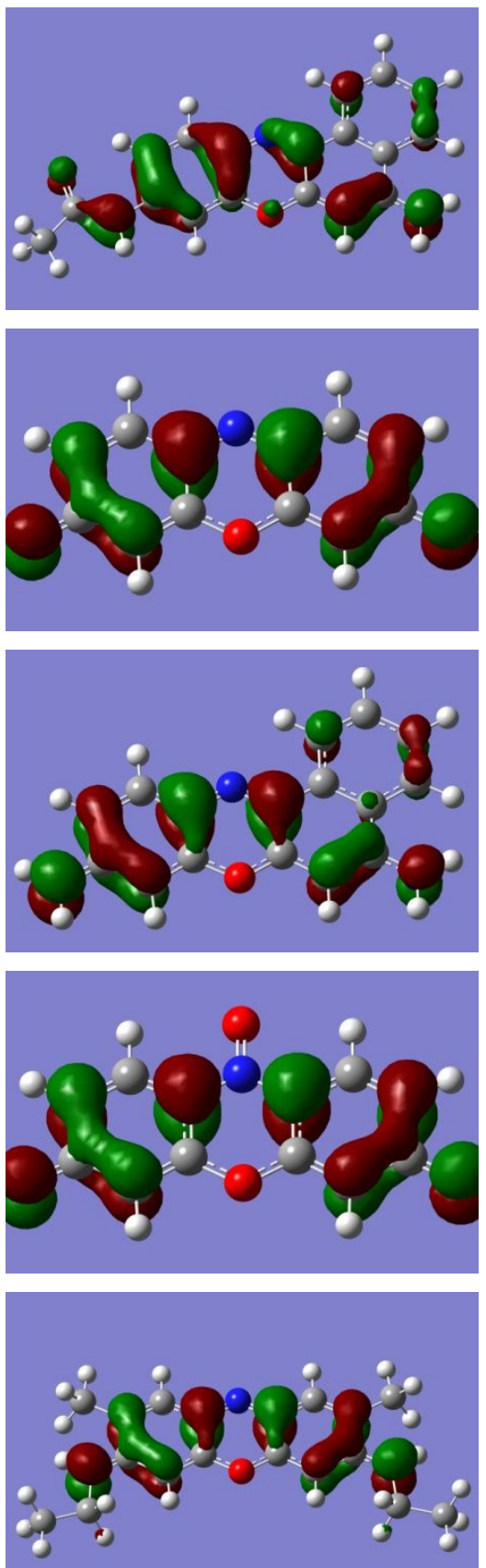

ey

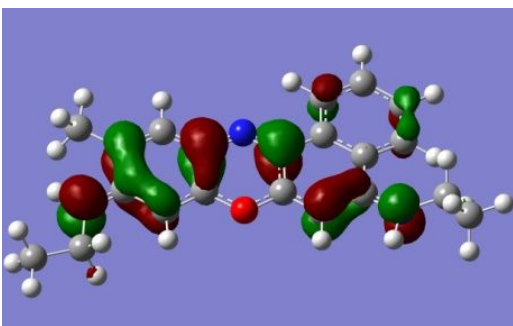

Virtual $\mathrm{MO}(\mathrm{s})$
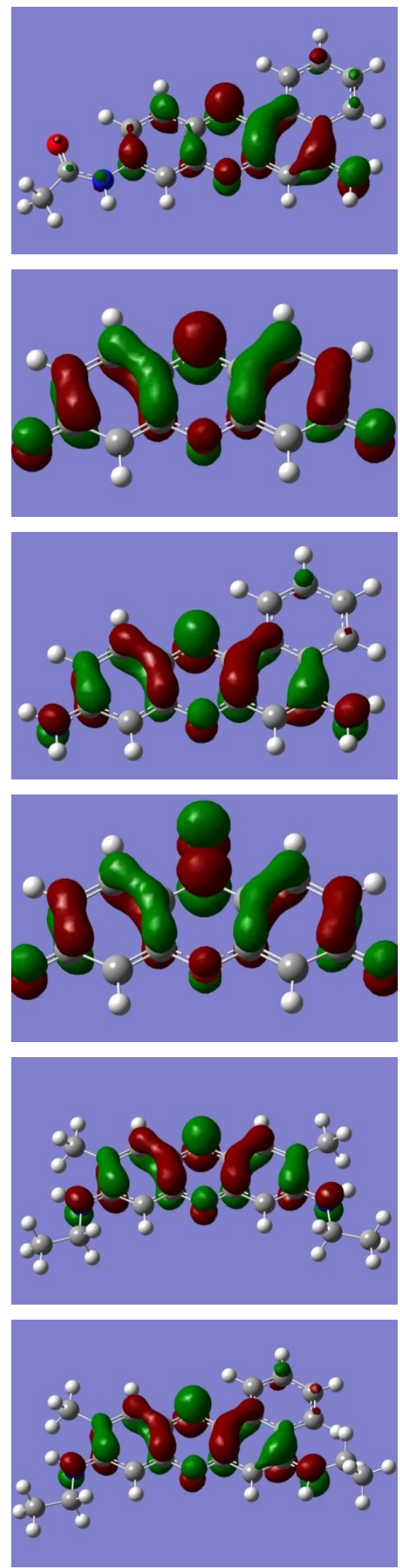
Table 4: Orbital pairs involved in the $\lambda_{\max }$ excitation for each dye. ${ }^{a}$ (continued)

7

8

9

10
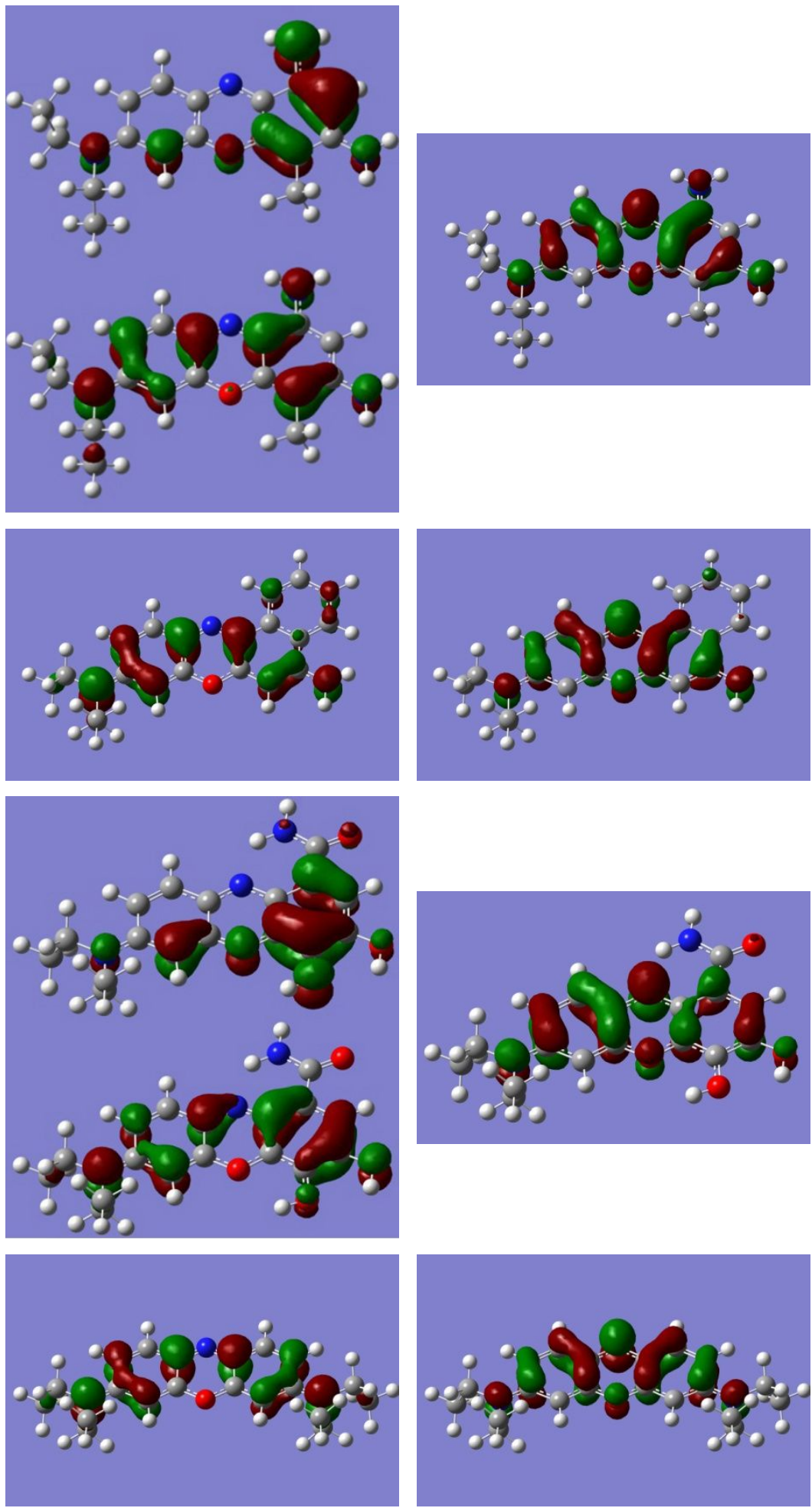

aAll orbitals are taken from the CPCM/B3LYP/6-311++G(d,p) single point calculation. Isovalue for surface $=0.04$.

tion energy in such cases which can be classified as problematic CT states. The calculated orbital overlaps in our series of oxazine dyes are all above the cut-off value, which is consistent with the general accuracy of the calculated excitation energies and suggests that the excitations do not fall into the category of being CT states. However, it is interesting to note that the 
overlap diagnostic does not cover all cases where TD-DFT fails to predict accurately the $\lambda_{\max }$. The most striking example of this is $\mathbf{1}$, which has strong orbital overlap and the largest error. However, as the authors of the diagnostic point out, "the test just states that low $\Lambda$ implies large errors, it does not preclude the possibility that high $\Lambda$ can also have large errors" [31].

\section{Conclusion}

In the current work we have investigated the ability of TD-DFT to predict the absorption spectra of a series of oxazine dyes and the effect of solvent on the accuracy of these predictions. Based on the results of this study, it is clear that for the series of oxazine dyes and accurate prediction of the excitation energy requires the inclusion of solvent. Implicit solvent included via a polarizable continuum approach was found to be sufficient in reproducing the excitation energies accurately in the majority of cases. Moreover, we found that the SMD solvent model gave more reliable results for our systems relative to the CPCM model, as implemented in Gaussian 09.

This study has also illustrated that for the oxazine dyes studied the principal excitation can be classified as an intramolecular CT excitation, based on the transition dipole moments of the excitations. Nonetheless, in all cases the inclusion of solvent reduces the error in the predicted excitation energy to $<0.3 \mathrm{eV}$ and in the majority of cases to $<0.1 \mathrm{eV}$.

\section{Experimental}

The commercially available oxazine dyes were used as supplied from Aldrich. Depending upon the solubility of the dye, deionized water was used as the solvent for all dyes. Counterions varied as summarized in Table 5. All absorption spectra were obtained using $1 \mathrm{~cm}$ cuvettes in a Cary $50 \mathrm{UV}$-vis spectrophotometer, scanning within the $200-800 \mathrm{~nm}$ range. Solutions of $10^{-4} \mathrm{~mol} \mathrm{dm}^{-3}$ were prepared in a $100 \mathrm{~cm}^{3}$ volumetric flask, and, if required, subsequently diluted by a factor of ten so as to obtain a maximum absorbance of less than 1 .

\section{Computational methods}

All structures were optimized in the gas-phase. For geometry optimizations, the B3LYP [32-37] level of theory with the $6-311++\mathrm{G}(\mathrm{d}, \mathrm{p})$ basis set $[38,39]$ was employed and no symmetry constraints were imposed. Time dependent density functional theory [13-17] (TD-DFT) single-point calculations were performed on the optimized structures to obtain the calculated $\lambda_{\max }$ values. The TD-DFT calculations were carried out in both the gas-phase and the aqueous phase. In order to evaluate the effect of the description of the solvent on the calculated spectra, both the conductor-like polarizable continuum model [40,41] (CPCM) and SMD [42] (Truhlar's new solvent model, which is dependent on the full electron density of the solute

\begin{tabular}{|ll}
\hline \multicolumn{2}{|l}{ Table 5: Counterions of each oxazine dye. } \\
\hline Dye & Counterion \\
\hline Nile blue & $\mathrm{SO}_{4}^{2-}$ \\
Brilliant cresyl blue & $\mathrm{ZnCl}_{4}^{2-}$ \\
Cresyl violet & $\mathrm{MeCOO}^{-}$ \\
Oxazine 1 & $\mathrm{ClO}_{4}^{-}$ \\
Oxazine 4 & $\mathrm{ClO}_{4}^{-}$ \\
Oxazine 170 & $\mathrm{ClO}_{4}^{-}$ \\
Celestine blue & $\mathrm{Cl}^{-}$ \\
Darrow red & $\mathrm{Cl}^{-}$ \\
Resazurin & $\mathrm{Na}^{+}$ \\
Resorufin & $\mathrm{Na}^{+}$ \\
& \\
\end{tabular}

without partitioning into partial charges) solvent models were employed within the TD-DFT calculations. The ability of different density functionals to accurately describe the excited states of the oxazine dye series was investigated by varying the functional employed in the single point TD-DFT calculations using the B3LYP optimized geometry of the molecule. The series of functionals investigated in this way includes B3LYP, CAM-B3LYP [43] (the coulomb attenuated version of the B3LYP functional which has been shown to provide a better description of CT states) [28], M06 [44], M06-L [45], and M06$2 \mathrm{X}$ [44] as the M06 suite of functionals have been shown to perform generally well for a range of molecular properties [46]. The M06-2X functional was included to examine the effect of an increased percentage of HF exchange on the ability of the functional to predict accurately the excitation energies as this has been shown to be beneficial in some cases [19]. All calculations were done within the Gaussian 09 program [47]. Finally, we have also employed the orbital overlap diagnostic of Tozer et al. in order to assess the CT character in the principal excited states [27].

\section{Acknowledgements}

TT thanks the Glasgow Centre of Physical Organic Chemistry for funding.

\section{References}

1. Mills, A.; Hazafy, D.; Parkinson, J.; Tuttle, T.; Hutchings, M. G. Dyes Pigm. 2011, 88, 149. doi:10.1016/j.dyepig.2010.05.015

2. Mills, A.; Hazafy, D.; Parkinson, J. A.; Tuttle, T.; Hutchings, M. G. J. Phys. Chem. A 2009, 113, 9575. doi:10.1021/jp9030927

3. Singh, M. K.; Pal, H.; Bhasikuttan, A. C.; Sapre, A. V. Photochem. Photobiol. 1998, 68, 32. doi:10.1111/j.1751-1097.1998.tb03249.x

4. Levine, A.; Schubert, M. J. Am. Chem. Soc. 1952, 74, 91. doi:10.1021/ja01121a021

5. Taylor, K. B. Stain Technol. 1961, 36, 73. doi:10.3109/10520296109113247 
6. Hintersteiner, M.; Enz, A.; Frey, P.; Jaton, A.-L.; Kinzy, W.; Kneuer, R.; Neumann, U.; Rudin, M.; Staufenbiel, M.; Stoeckli, M.; Wiederhold, K.-H.; Gremlich, H.-U. Nat. Biotechnol. 2005, 23, 577. doi:10.1038/nbt1085

7. Knemeyer, J.-P.; Marmé, N.; Sauer, M. Anal. Chem. 2000, 72, 3717. doi:10.1021/ac000024o

8. Vogelsang, J.; Cordes, T.; Forthmann, C.; Steinhauer, C.; Tinnefeld, P. Proc. Natl. Acad. Sci. U. S. A. 2009, 106, 8107.

doi:10.1073/pnas.0811875106

9. Deye, J. F.; Berger, T. A.; Anderson, A. G. Anal. Chem. 1990, 62, 615. doi:10.1021/ac00205a015

10. Ghanadzadeh, A.; Zeini, A.; Kashef, A.; Moghadam, M. Spectrochim. Acta, Part A: Mol. Biomol. Spectrosc. 2009, 73, 324. doi:10.1016/j.saa.2009.02.029

11. Tajalli, H.; Gilani, A. G.; Zakerhamidi, M. S.; Tajalli, P. Dyes Pigm. 2008, 78, 15. doi:10.1016/j.dyepig.2007.10.002

12. Tuck, P. O.; Mawhinney, R. C.; Rappon, M. Phys. Chem. Chem. Phys. 2009, 11, 4471. doi:10.1039/b902528f

13. Bauernschmitt, R.; Ahlrichs, R. Chem. Phys. Lett. 1996, 256, 454. doi:10.1016/0009-2614(96)00440-X

14. Casida, M. E.; Jamorski, C.; Casida, K. C.; Salahub, D. R. J. Chem. Phys. 1998, 108, 4439. doi:10.1063/1.475855

15. Marques, M. A. L.; Gross, E. K. U. Annu. Rev. Phys. Chem. 2004, 55 , 427. doi:10.1146/annurev.physchem.55.091602.094449

16. Runge, E.; Gross, E. K. U. Phys. Rev. Lett. 1984, 52, 997. doi:10.1103/PhysRevLett.52.997

17. Stratmann, R. E.; Scuseria, G. E.; Frisch, M. J. J. Chem. Phys. 1998, 109, 8218. doi:10.1063/1.477483

18. Tozer, D. J.; Handy, N. C. J. Chem. Phys. 1998, 109, 10180. doi:10.1063/1.477711

19. Goerigk, L.; Grimme, S. J. Chem. Phys. 2010, 132, 184103. doi:10.1063/1.3418614

20. Goerigk, L.; Moellmann, J.; Grimme, S. Phys. Chem. Chem. Phys. 2009, 11, 4611. doi:10.1039/b902315a

21. Jacquemin, D.; Perpète, E. A.; Ciofini, I.; Adamo, C.; Valero, R.; Zhao, Y.; Truhlar, D. G. J. Chem. Theory Comput. 2010, 6, 2071. doi:10.1021/ct100119e

22. Jacquemin, D.; Wathelet, V.; Perpète, E. A.; Adamo, C. J. Chem. Theory Comput. 2009, 5, 2420. doi:10.1021/ct900298e

23. Silva, M. R.; Schreiber, M.; Sauer, S. P. A.; Thiel, W. J. Chem. Phys. 2008, 129, 104103. doi:10.1063/1.2973541

24. Anand, S.; Schlegel, H. B. Mol. Phys. 2006, 104, 933. doi:10.1080/00268970500418042

25. Dreuw, A.; Fleming, G. R.; Head-Gordon, M. J. Phys. Chem. B 2003, 107, 6500. doi:10.1021/jp034562r

26. Fabian, J. Theor. Chem. Acc. 2001, 106, 199. doi:10.1007/s002140100250

27. Peach, M. J. G.; Benfield, P.; Helgaker, T.; Tozer, D. J. J. Chem. Phys. 2008, 128, 044118. doi:10.1063/1.2831900

28. Peach, M. J. G.; Helgaker, T.; Sałek, P.; Keal, T. W.; Lutnæs, O. B.; Tozer, D. J.; Handy, N. C. Phys. Chem. Chem. Phys. 2006, 8, 558. doi:10.1039/b511865d

29. Tozer, D. J.; Amos, R. D.; Handy, N. C.; Roos, B. O.; Serrano-Andres, L. Mol. Phys. 1999, 97, 859. doi:10.1080/00268979909482888

30. Jamorski, C.; Foresman, J. B.; Thilgen, C.; Lüthi, H.-P. J. Chem. Phys. 2002, 116, 8761. doi:10.1063/1.1465404

31. Peach, M. J. G.; Le Sueur, C. R.; Ruud, K.; Guillaume, M.; Tozer, D. J. Phys. Chem. Chem. Phys. 2009, 11, 4465. doi:10.1039/b822941d
32. Becke, A. D. Phys. Rev. A 1988, 38, 3098. doi:10.1103/PhysRevA.38.3098

33. Becke, A. D. J. Chem. Phys. 1993, 98, 5648. doi:10.1063/1.464913

34. Hertwig, R. H.; Koch, W. Chem. Phys. Lett. 1997, 268, 345. doi:10.1016/S0009-2614(97)00207-8

35. Lee, C. T.; Yang, W. T.; Parr, R. G. Phys. Rev. B 1988, 37, 785 doi:10.1103/PhysRevB.37.785

36. Stephens, P. J.; Devlin, F. J.; Chabalowski, C. F.; Frisch, M. J. J. Phys. Chem. 1994, 98, 11623. doi:10.1021/j100096a001

37. Vosko, S. H.; Wilk, L.; Nusair, M. Can. J. Phys. 1980, 58, 1200. doi:10.1139/p80-159

38. Hariharan, P. C.; Pople, J. A. Theor. Chim. Acta 1973, 28, 213. doi:10.1007/BF00533485

39. Krishnan, R.; Binkley, J. S.; Seeger, R.; Pople, J. A. J. Chem. Phys. 1980, 72, 650. doi:10.1063/1.438955

40. Barone, V.; Cossi, M. J. Phys. Chem. A 1998, 102, 1995. doi:10.1021/jp9716997

41. Cossi, M.; Rega, N.; Scalmani, G.; Barone, V. J. Comput. Chem. 2003, 24, 669. doi:10.1002/jcc.10189

42. Marenich, A. V.; Cramer, C. J.; Truhlar, D. G. J. Phys. Chem. B 2009, 113, 6378. doi:10.1021/jp810292n

43. Yanai, T.; Tew, D. P.; Handy, N. C. Chem. Phys. Lett. 2004, 393, 51. doi:10.1016/j.cplett.2004.06.011

44. Zhao, Y.; Truhlar, D. G. Theor. Chem. Acc. 2008, 120, 215. doi:10.1007/s00214-007-0310-x

45. Zhao, Y.; Truhlar, D. G. J. Chem. Phys. 2006, 125, 194101. doi:10.1063/1.2370993

46. Zhao, Y.; Truhlar, D. G. Acc. Chem. Res. 2008, 41, 157. doi:10.1021/ar700111a

47. Gaussian 09, A.02; Gaussian, Inc.: Wallingford, CT, 2009.

\section{License and Terms}

This is an Open Access article under the terms of the Creative Commons Attribution License (http://creativecommons.org/licenses/by/2.0), which permits unrestricted use, distribution, and reproduction in any medium, provided the original work is properly cited.

The license is subject to the Beilstein Journal of Organic Chemistry terms and conditions:

(http://www.beilstein-journals.org/bjoc)

The definitive version of this article is the electronic one which can be found at: doi:10.3762/bjoc.7.56 